\title{
SELECCIÓN DE ESPECIES LEÑOSAS POR VACUNOS EN SILVO- PASTOREO DE UN BOSQUE SEMICADUCIFOLIO EN VENEZUELA
}

\author{
WOODY SPECIES SELECTIVITY BY CATTLE IN SILVOPASTORAL CONDITIONS INA \\ SEMI-DECIDUOUS FOREST IN VENEZUELA
}

\author{
Ojeda, A. ${ }^{*}$, Obispo, N. ${ }^{2}$, Canelones, C.E. ${ }^{1}$ y Muñoz, D. ${ }^{1}$ \\ ${ }^{1}$ Instituto de Producción Animal. Facultad de Agronomía. Universidad Central de Venezuela. Maracay. \\ Venezuela. *ajojeda99@yahoo.com \\ ${ }^{2}$ Instituto Nacional de Investigaciones Agrícolas. Centro Nacional de Investigaciones Agropecuarias. \\ Maracay. Aragua. Venezuela.
}

\section{Palabras clave adicionales}

Análisis químico. Composición botánica. Taninos.

\section{RESUMEN}

Para determinar la selección de plantas leñosas por vacunos en silvopastoreo de un bosque semicaducifolio tropical (BSCT), se evaluaron fragmentos epidérmicos en muestras de heces de 14 vacunos machos Brahman x Holstein (390,4 \pm $18,0 \mathrm{~kg}$ peso vivo). Los animales pastaron durante 91 días sobre 81 ha localizadas en el estado Portuguesa, Venezuela, donde 50 ha correspondieron a un BSCT y el resto a pastizales con predomino de Cynodon nlemfuensis, Sporobolus indicus, Cyperus sp. y Axonopus sp. Empleando la técnica de puntos en cuadrante sobre transectas, en el bosque se identificaron 22 especies de plantas leñosas, agrupadas en 11 familias botánicas con $40,9 \%$ de las especies dentro de la familia Fabaceae. La especie Guazuma ulmifolia tuvo la mayor dominancia relativa $(60,4 \%)$, acumulando conjuntamente con Pithecellobium lanceolatum y Samanea saman el $81,1 \%$ de este parámetro. Estas mismas especies mostraron los mayores índices de valor de importancia $(162,6$ 24,8 y 26,5 respectivamente). Las Poaceae presentaron la mayor frecuencia relativa de aparición de fragmentos epidémicos en heces con el $49,6 \%$, mientras el conjunto de plantas leñosas presentó un $46,2 \%$, del cual las Fabaceae alcanzaron el $80,7 \%$. Las especies más seleccionadas fueron Inga laurina, Machaerium humboldtianum, $S$. saman y Sida acuta, con un Índice de Ivlev medio de 0,60 $\pm 0,09$. La composición química del follaje de las plantas leñosas sugiere un patrón de

Recibido: 27-4-11. Aceptado: 22-12-11.

\section{AdDitionAL KEYWORDS}

Chemical analyses. Botanical composition. Tannins.

selección ajustado a la oferta de nutrientes de cada especie, resaltando el valor estratégico del BSCT en sistemas de silvopastoreo en ambientes tropicales.

\section{SUMMARY}

The woody plant palatability for cattle in a silvopastoral system on a semi-deciduous tropical forest (SDTF) was evaluated. Epidermal fragments in fecal samples from 14 male cattle Brahman $\mathrm{x}$ Holstein (390.4 $\pm 18.0 \mathrm{~kg}$ live weight) grazing during 91 days in 81 ha located in Portuguesa state, Venezuela were evaluated. SDTF was present in 50 ha; the rest of grassland, was mainly composed by Cynodon nlemfuensis, Sporobolus indicus, Cyperus sp. and Axonopus sp. Using the point quarter method on transects, 22 woody plant species were identified they were grouped into 11 botanical families, with $40.9 \%$ of species belonging to the Fabaceae family. Guazuma ulmifolia had the greater relative dominancy $(60.4 \%)$, and together with Pithecellobium lanceolatum and Samanea saman reached the $81.1 \%$. These same species showed the higher importance value index (162.6, 24.8 and 26.5 respectively). Poaceae had the higher relative frequency $(49.6 \%)$ for epidermal fragments in feces; total woody plants reached $46.2 \%$ (Fabaceae: $80.7 \%$ ). The most selected species were Inga laurina, Machaerium humboldtianum, S. saman and Sida acuta, (Ivlev 


\section{OJEDA, OBISPO, CANELONES Y MUÑOZ}

index $=0.60 \pm 0.09$ ). The chemical composition of woody plants foliage suggest a selectivity profile adjusted to the nutrient profile offered by each species. These findings highlight the strategic value of SDTF in silvopastoral systems in tropical environments.

\section{INTRODUCCIÓN}

En América tropical la actividad ganadera es fundamentalmente pastoril, con rebaños distribuidos sobre grandes extensiones de sabanas dominadas por pastizales naturales. En el caso de Venezuela, se estima que se cuenta con unas $29 \times 10^{6}$ ha de sabanas, asociadas en un $80 \%$ a formaciones de bosque seco tropical (Baldizán y Chacón, 2007), con marcada estacionalidad en las precipitaciones que incide negativamente en la oferta y calidad de la materia seca proveniente de plantas herbáceas. Ante esta situación, a medida que avanza el periodo seco los granjeros promueven el ingreso de sus rebaños vacunos a formaciones boscosas, en una modalidad desarrollada de modo empírico con el objeto de garantizar un incremento en la cantidad y calidad de la biomasa forrajera.

Para aprovechar las ventajas relativas de esta estrategia de silvopastoreo estacional de bosques tropicales, se requiere generar información acerca de los hábitos alimentarios del animal, así como la valoración química y nutricional de las especies leñosas de valor forrajero presentes en dichos sistemas, lo que permitirá estructurar programas de manejo sustentables que den un valor agregado a dichos bosques, para promover que el productor sea parte activa de los planes de preservación de tales ecosistemas. A nivel nacional, se ha avanzado en estudios de algunas plantas leñosas de valor forrajero y evaluaciones de sistemas silvopastoriles en formaciones de bosque seco caducifolio (Benezra et al., 2003; Baldizán et al., 2006; García et al., 2006), no existiendo información disponible sobre la selección de plantas leñosas por vacunos en bosques secos semicaduci- folios, localizados en áreas de amplio desarrollo de la actividad ganadera del trópico americano.

El objetivo del presente estudio fue identificar y caracterizar químicamente las plantas leñosas seleccionadas por vacunos en silvopastoreo en un bosque semicaducifolio tropical en Venezuela, para generar información básica del valor forrajero potencial de algunas especies del sotobosque.

\section{MATERIAL Y MÉTODOS}

La experiencia se desarrolló en un lapso de 91 días, durante los meses de enero a marzo 2008 en la Estación Experimental San Nicolás ( $8^{\circ} 49^{\prime} 58^{\prime \prime}$ latitud Norte y $69^{\circ} 48^{\prime}$ $00^{\prime \prime}$ longitud Oeste), adscrita a la Universidad Central de Venezuela. Se seleccionó una superficie de 81 ha, dentro de la cual se localizan 50 ha de un bosque semicaducifolio tropical de bajo nivel de intervención. Las restantes 31 ha estuvieron conformadas por vegetación herbácea en crecimiento vegetativo constituida por Cynodon nlemfuensis, Sporobolus indicus, Axonopus sp., Mimosa púdica e Hyptissu aveolens, entre otras; con un rendimiento promedio durante la experiencia de $969,3 \pm 28,6 \mathrm{~kg} \mathrm{MS} /$ ha y una relación hoja:tallo aproximada de $0,53 \pm 0,15$. Previo al desarrollo del estudio el potrero presentó un descanso en la rotación de 55 días, realizando las evaluaciones en la época seca. Durante la fase experimental, la unidad climatológica de la estación registró $62,1 \mathrm{~mm}$ de precipitación, $26,9 \pm 0,8^{\circ} \mathrm{C}$ de temperatura media diaria y humedad relativa de $56 \pm 4,9 \%$.

Para determinar la composición botánica de la flora leñosa en el bosque semicaducifolio, y en función a la diversidad de la cubierta vegetal, se realizaron 12 transectas de 100 a $200 \mathrm{~m}$ de longitud y $2-4 \mathrm{~m}$ de ancho, con distancia entre puntos de muestreo de $10 \mathrm{~m}$, de acuerdo a la técnica de puntos en cuadrante (López et al., 1998). Del estrato susceptible de ramoneo por vacunos adultos (altura máxima de $2 \mathrm{~m}$ ), men- 


\section{SELECCIÓN DE ESPECIES LEÑOSAS POR VACUNOS}

sualmente se tomaron muestras de hojas, foliolos, estructuras reproductivas y tallos con diámetro inferior a $6 \mathrm{~mm}$ hasta obtener $900 \mathrm{~g}$ de material fresco/planta, eligiendo en cada ocasión 10 plantas leñosas por especie seleccionada por los animales, observando que las mismas presentasen edad y estado fenológico similares.

Se emplearon 14 vacunos, machos enteros mestizos Brahman x Holstein de 390,4 \pm $18,0 \mathrm{~kg}$ de peso vivo (PV), manejados bajo pastoreo continuo con disponibilidad ad libitum de agua y una mezcla comercial de sales y minerales con $20 \% \mathrm{Ca}, 10 \% \mathrm{P}, 9 \% \mathrm{Na}$, $0,5 \% \mathrm{Mg}, 0,6 \% \mathrm{~S}, 0,5 \% \mathrm{Zn}, 0,25 \% \mathrm{Mn}, 0,25 \%$ $\mathrm{Cu}, 0,15 \%$ F, 20 ppm Co, 20 ppm Se y 80 ppm I. Con el objeto de permitir que la selectividad de los animales en estudio fuese en condiciones de carga animal usuales para sabanas naturales, y en función a la biomasa graminiforme presente, se emplearon animales adicionales para garantizar una presión de pastoreo de $6 \mathrm{~kg} \mathrm{MS} / 100 \mathrm{~kg}$ PV y una variación de peso de $400 \pm 35 \mathrm{~g} / \mathrm{anima} /$ día Quince días previos al inicio de la evaluación, todos los animales fueron desparasitados con clorhidrato de levamisol $(1 \mathrm{~mL} / 50 \mathrm{~kg}$ peso) y se les aplicó $1 \mathrm{~mL} / 100 \mathrm{~kg}$ de PV de un complejo multivitamínico $(500000 \mathrm{UI} / \mathrm{mL}$ vit. A, $75000 \mathrm{UI} / \mathrm{mL}$ vit. $\mathrm{D}_{3}$ y $50 \mathrm{UI} / \mathrm{mL}$ vit. E).

La composición estructural de la dieta fue estimada a partir de la evaluación de muestras de heces colectadas cada 15 días entre las 8:00 y 10:00 horas, de forma individual y directamente del recto. Una vez colectadas, las muestras fueron depositadas en bolsas plásticas y almacenadas a $-18^{\circ} \mathrm{C}$ hasta su deshidratación en estufa con ventilación forzada a $65^{\circ} \mathrm{C}$ durante 48 horas. A continuación, las muestras fueron molidas en criba de $1 \mathrm{~mm} \varnothing$ y tomados $2 \mathrm{~g}$ los cuales se sometieron a un proceso de rehidratación con alcohol etílico al 50\% $\mathrm{v} / \mathrm{v}$, tamizado $(180 \mu)$ y remoción de los pigmentos vegetales con hipoclorito de sodio, siguiendo la rutina descrita por Holeckek (1982). Posteriormente, se les adicionaron $20 \mathrm{~mL}$ de glicerina al $10 \%$ y 4 gotas de azul de toloudina al $1 \%$, realizando el montaje semipermanente a razón de 3 láminas de $24 \times 24 \mathrm{~mm}$ por cada muestra. La evaluación de fragmentos epidérmicos se efectuó con un microscopio binocular con magnificación de 400X, empleando vistas transversales de $2 \mathrm{~mm}$ de ancho, $60 \mathrm{~mm}$ de longitud y $3 \mathrm{~mm}$ entre transversales. Para todas las plantas leñosas presentes en el área bajo estudio, al inicio de la fase experimental fueron desarrollados patrones fotográficos digitales de referencia empleando como elementos de identificación la caracterización morfológica de tricomas, estomas, cristales y células epidérmicas típicas.

De acuerdo a lo propuesto por MullerDumbois y Ellemberg (1974), para las plantas leñosas presentes en el área de bosque se estimó la densidad relativa como el número de individuos de una especie sobre la totalidad de individuos para todas las especies, la dominancia relativa como el resultado de multiplicar el número de individuos de una especie por el diámetro de su tallo a 1,3 $\mathrm{m}$ de altura (dominancia absoluta) en función a la sumatoria de la dominancia absoluta de todas las especies, y la frecuencia relativa considerando el número de puntos de muestreo sobre las transectas donde aparece cada especie, sobre la totalidad de puntos donde aparecen el resto de especies evaluadas. El índice de valor de importancia (IVI), parámetro adimensional que estima el éxito ecológico relativo de una especie dentro de una comunidad vegetal (Barbour et al., 1999), se determinó empleando la siguiente ecuación:

$\mathrm{IVI}=\Sigma[$ Densidad $(\%)+$ Frecuencia $(\%)+$ Dominancia $(\%)]$

El grado de selección de especies arbustivas y arbóreas para cada planta fue estimado a través del índice de selectividad (IS) de Ivlev citado por Lechowicz (1982), considerando valores entre + 1 (máxima preferencia) y -1 (rechazo total), mientras registros cercanos a cero indican (en función de 
la cantidad de fragmentos epidérmicos en heces) que la especie vegetal fue seleccionada en relación directa con su densidad en el área de pastoreo. A tales fines para cada planta se empleó la fórmula siguiente:

$$
\mathrm{IS}=\frac{\text { Fragmentos epidérmicos }(\%)-\mathrm{IVI}(\%)}{\text { Fragmentos epidérmicos }(\%)+\mathrm{IVI}(\%)}
$$

Muestras compuestas de $400 \mathrm{~g}$ del follaje de las plantas leñosas seleccionadas por los animales fueron deshidratadas hasta peso constante en estufa con ventilación forzada a $65^{\circ} \mathrm{C}$ para determinar la materia seca (AOAC, 1990). Para los análisis bromatológicos y cuantificación de metabolitos secundarios, $400 \mathrm{~g}$ de muestra fueron deshidratados a temperatura ambiente en ausencia de luz durante cinco días. Posteriormente fueron molidos a un tamaño de partícula de $1 \mathrm{~mm}$, y almacenados en envases de color ámbar hasta la realización de los análisis de laboratorio.

Se determinaron los contenidos de proteína bruta $\left(\mathrm{PB}, \mathrm{N}^{*} 6,25\right)$ y extracto etéreo (EE), de acuerdo a AOAC (1990), calcio (Fick et al., 1979) y fósforo (Fiske $\mathrm{y}$ Subarrow, 1925). Las determinaciones de fibra en detergente neutro (FDN) y ácido (FDA), celulosa y lignina se efectuaron según Van Soest et al. (1991), con la adición de sulfito de sodio a la solución de detergente neutro para remover parcialmente los complejos taninos-proteína (Terril et al., 1994). La cuantificación de los fenoles totales extractables (FT) se realizó mediante el método de Folin-Ciocalteu modificado por Makkar (2001), expresando los resultados como equivalentes (Eat) de ácido tánico (Lab. Merck $\left.{ }^{\circledR}\right)$. Los taninos condensados extractables (TC) se determinaron usando $\mathrm{nButanol} / \mathrm{HCl} / \mathrm{Fe}^{3+}$ (Porter et al., 1986), expresando los resultados como equivalentes de leucocianidinas (Eleu) en base seca. La astringencia se estimó mediante el ensayo de difusión radial (Hagerman, 1987), empleando una solución con base de agarosa
(Sigma ${ }^{\circledR}$ A-6013/ Tipo I: Low EEO) en buffer de acetato y seroalbúmina bovina (Sigma A3350), y expresando los resultados en gramos Eat en porcentaje de la materia seca.

La frecuencia relativa de aparición de fragmentos epidérmicos en heces fue analizada en forma no paramétrica empleando la prueba de Kruskal-Wallis (Steel y Torrie, 1985), mientras la composición química se evaluó a través de un diseño completamente aleatorizado, asumiendo cada especie como un tratamiento, y efectuando las comparaciones entre medias mediante la prueba de comparación múltiple de Tukey. La relación entre la selectividad del follaje de las especies leñosas y su composición química se estableció mediante el coeficiente de correlación de Pearson.Toda la información generada se evaluó con el empleo del paquete estadístico SAS (SAS, 1994).

\section{RESULTADOSYDISCUSIÓN}

En la tabla I se presenta la composición botánica del bosque semicaducifolio. Se identificaron 22 especies de plantas leñosas agrupadas en 11 familias botánicas, de las cuales un $40,9 \%$ pertenecieron a la familia Fabaceae, ubicándose luego las familias Malvaceae, Moraceae y Polygonaceae, cada una con $9,1 \%$ del total de especies leñosas presentes. Comparada con otras formaciones boscosas de valor actual o potencial para el desarrollo de sistemas silvopastoriles, esta comunidad semicaducifolia evidenció una importante diversidad florística, donde destaca la participación de Fabáceas (Grela, 2003; Benezra et al., 2003; Baldizán et al., 2006; Miliani et al., 2008). Además de su valor nutricional al rumiante, las Fabáceas aportan nitrógeno y participan en el reciclaje de Ca y $\mathrm{P}$ en el suelo, por lo que poseen un valor agregado como parte de la biomasa del bosque con potencial forrajero (Rosales, 1998).

Con una densidad de 463 plantas/ha, cuatro especies (Guazuma ulmifolia, Pithecellobium lanceolatum, Samanea 


\section{SELECCIÓN DE ESPECIES LEÑOSAS POR VACUNOS}

Tabla I. Composición botánica e indicadores de importancia de especies leñosas del bosque semicaducifolio tropical. (Botanical composition and importance indicators of the woody species from semi-deciduous tropical forest).

\begin{tabular}{|c|c|c|c|c|c|}
\hline \multirow[b]{2}{*}{ Familia } & \multirow[b]{2}{*}{ Nombre científico } & \multirow[b]{2}{*}{$\begin{array}{c}\text { densidad } \\
(\%)\end{array}$} & \multicolumn{2}{|c|}{ Indicadores } & \multirow[b]{2}{*}{ IVI } \\
\hline & & & $\begin{array}{c}\text { frecuencia } \\
(\%)\end{array}$ & $\begin{array}{c}\text { dominancia } \\
(\%)\end{array}$ & \\
\hline Arecaceae & Acrocomia aculeata & 0,4 & 0,5 & 0,5 & 1,4 \\
\hline Boraginaceae & Rochefortia spinosa & 3,0 & 0,9 & 1,6 & 5,5 \\
\hline Chrysobalanaceae & Parinari campestris & 0,3 & 1,9 & 1,1 & 3,3 \\
\hline \multirow[t]{9}{*}{ Fabaceae } & Enterolobium cyclocarpus & 0,4 & 3,9 & 0,3 & 4,6 \\
\hline & Erithrina fusca & 1,7 & 1,9 & 2,4 & 6 \\
\hline & Inga laurina & 0,5 & 1,0 & 0,2 & 1,7 \\
\hline & Machaerium humboldtianum & 0,7 & 1,1 & 0,5 & 2,3 \\
\hline & Pithecellobium lanceolatum & 6,4 & 11,2 & 7,2 & 24,8 \\
\hline & Pterocarpus sp. & 1,8 & 1,9 & 1,3 & 5 \\
\hline & Samanea saman & 6,2 & 6,8 & 13,5 & 26,5 \\
\hline & Senna pallida & 1,3 & 2,9 & 0,6 & 4,8 \\
\hline & Senna sp. & 0,6 & 1,8 & 0,3 & 2,7 \\
\hline Poaceae & Guadua angustifolia & 6,0 & 5,8 & 1,9 & 13,7 \\
\hline \multirow[t]{2}{*}{ Malvaceae } & Hibiscus sp. & 1,3 & 0,2 & 0,2 & 1,7 \\
\hline & Sida acuta & 1,3 & 0,9 & 0,3 & 2,5 \\
\hline \multirow[t]{2}{*}{ Moraceae } & Morus sp. & 0,4 & 0,5 & 0,1 & 1 \\
\hline & Cecropia peltata & 1,1 & 2,9 & 1,3 & 5,3 \\
\hline Rubiaceae & Genipa americana & 0,4 & 0,3 & 0,1 & 0,8 \\
\hline Piperaceae & Piper sp. & 0,2 & 0,5 & 0,1 & 0,8 \\
\hline \multirow{2}{*}{ Polygonaceae } & Coccoloba caracasana & 0,5 & 5,8 & 0,3 & 6,6 \\
\hline & Triplaris caracasana & 0,2 & 4,8 & 0,2 & 5,2 \\
\hline Sterculiaceae & Guazuma ulmifolia & 61,6 & 40,6 & 60,4 & 162,6 \\
\hline No identificadas & & 3,7 & 1,9 & 5,6 & 11,2 \\
\hline
\end{tabular}

IVI: Índice de valor de importancia.

saman y Guadua angustifolia) acumularon el $80,2 \%$ del total de las plantas leñosas identificadas, mientras que un $3,7 \%$ de las especies leñosas no pudieron ser identificadas botánicamente, lo que representó un valor situado dentro de los márgenes señalados en investigaciones similares (Grela, 2003; Valero et al., 2005). El estudio reveló que 5 especies ( $G$. ulmifolia, $P$. lanceolatum, S. saman, G. angustifolia y Coccoloba caracasana,) se localizaron en el 70,2\% de los puntos evaluados en las transectas, lo cual indica su amplia distribución. La especie G. ulmifolia mostró una clara dominancia relativa $(60,4 \%)$ dentro de la comunidad, no sólo producto de su elevada densidad, sino también por el grado de desarrollo de los ejemplares localizados. Esta especie, conjuntamente con $S$. saman y P. lanceolatum, acumularon una dominancia relativa de $81,1 \%$.

G. ulmifolia presentó el mayor registro para el IVI $(162,6)$, seguida en importancia por las especies $S$. saman y $P$. lanceolatum $(25,7 \pm 1,2)$, acumulando en su conjunto estas especies el 71,2\% de dicho indicador. Esto sugiere el valor forrajero potencial de estas leñosas, ya que el IVI se asocia al consumo voluntario, en virtud de la ausencia de limitantes físicas o químicas a la ingesta. 
La distribución espacial influye de modo decisivo en la oportunidad que tienen los animales en pastoreo de tener contacto con una especie (Provenza y Balph, 1988). De los fragmentos evaluados en heces el 49,6\% correspondió a poáceas (tabla II), y un 46,2\% a 10 plantas leñosas. El restante $4,2 \%$ no pudo ser identificado. E1 80,7\% de los fragmentos de plantas leñosas se asoció a miembros de la familia Fabaceae, siendo $S$. saman la especie más seleccionada $(26,4 \% ; \mathrm{p}<0,05)$, mientras que $C$. caracasana la menos preferida $(0,8 \% ; \mathrm{p}<0,05)$.

La amplia selección de poáceas y fabáceas por parte de vacunos en pastoreo ha sido documentada en diversos estudios (Mayer et al., 2003; Pereira et al., 2003; Benezra et al., 2003; Rutter, 2006). Si bien los mayores componentes de la ración tienden a mantener su prevalencia bajo diferentes circunstancias de manejo, variaciones en la composición botánica dietaria son influenciadas por las condiciones climáticas que regulan la variabilidad temporal y espacial de la oferta forrajera y algunos factores intrínsecos de la planta, tales como características nutricionales, arquitectura, presencia de espinas y pubescencia de las hojas, entre otros; además de edad y estado fisiológico del animal, intensidad de pastoreo, experiencias alimentarias preliminares, aprendizaje social (individual y colectivo) y respuesta neofóbica, entre otros (Marquardt et al., 2009). A lo que se puede añadir la existencia de un patrón circadiano, con el mayor consumo de fabáceas por la mañana (Rutter, 2006).

Los valores del IS (tabla II) indican que los animales mostraron la mayor preferencia por S. acuta, una Malvaceae tradicionalmente definida como maleza arbustiva en áreas de pastizales, pero que a la luz de estos resultados debe ser considerada por su valor forrajero potencial. Luego aparecen plantas de la familia Fabaceae (I. laurina, S. saman y $M$. humboldtianum) con un IS de 0,51 \pm 3,06 , mientras que de acuerdo a este indicador, los animales evidenciaron rechazo por
Tabla II. Fragmentos epidérmicos vegetales en heces e indice de selectividad de Ivlev en especies del bosque semicaducifolio tropical. (Epidermal fragments in the feces and Ivlev selectivity index for species from semi-deciduous tropical forest).

\begin{tabular}{lcc}
\hline Identificación & $\begin{array}{c}\text { Fragmentos } \\
\text { epidérmicos }(\%)\end{array}$ & $\begin{array}{c}\text { Índice } \\
\text { de Ivlev }\end{array}$ \\
\hline R. spinosa & $2,81^{\mathrm{c}}$ & $0,19^{\mathrm{c}}$ \\
E. cyclocarpus & $2,13^{\mathrm{c}}$ & $0,17^{\mathrm{c}}$ \\
I. laurina & $2,03^{\mathrm{c}}$ & $0,54^{\mathrm{ab}}$ \\
M. humboldtianum & $4,26^{\mathrm{c}}$ & $0,48^{\mathrm{b}}$ \\
P. lanceolatum & $1,44^{\mathrm{c}}$ & $-0,71^{\mathrm{d}}$ \\
S. saman & $26,43^{\mathrm{b}}$ & $0,50^{\mathrm{b}}$ \\
Senna sp. & $1,11^{\mathrm{c}}$ & $0,10^{\mathrm{c}}$ \\
S. acuta & $4,07^{\mathrm{c}}$ & $0,67^{\mathrm{a}}$ \\
G. ulmifolia & $1,46^{\mathrm{c}}$ & $-0,75^{\mathrm{d}}$ \\
C. caracasana & $0,75^{\mathrm{d}}$ & $-0,52^{\mathrm{d}}$ \\
Poaceae & $49,62^{\mathrm{a}}$ & \\
No identificadas & $4,21^{\mathrm{c}}$ & \\
& & \\
Error estándar & $2,4^{*}$ & $0,12^{*}$ \\
\hline
\end{tabular}

abcdMedias con superíndices desiguales en columnas difieren estadísticamente $\left({ }^{*} p<0,05\right)$.

G. ulmifolia, P. lanceolatum y C. caracasana $(-0,73 \pm 0,22)$.

La figura 1 presenta la evolución de los fragmentos epidérmicos en heces en contraste con la biomasa herbácea presente en el área de pastoreo. En general, se observan pocas variaciones en los valores medios de los fragmentos epidérmicos de poáceas y plantas leñosas debido a que el manejo de la experiencia contempló una oferta de biomasa herbácea ajustada a una presión constante de pastoreo, tal como se menciona previamente. La mayor variabilidad asociada a plantas leñosas al inicio de la experiencia (enero) puede deberse al impacto de la fase de adaptación al manejo, donde la exploración y selección es condicionada por la presencia de compuestos, aspectos organolépticos y de la fisiología animal con características disuasivas o estimuladoras del consumo (Marquardt et al., 2009). 


\section{SELECCIÓN DE ESPECIES LEÑOSAS POR VACUNOS}

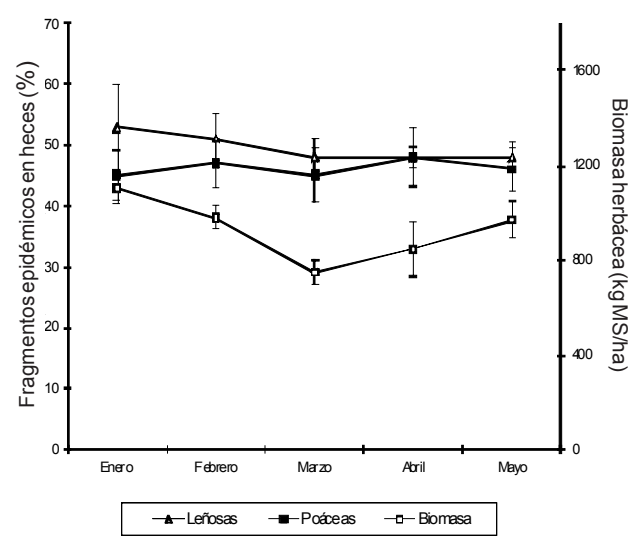

Figura 1. Dinámica de la biomasa herbácea y los fragmentos epidémicos de especies del bosque semicaducifolio tropical, en heces. (Dynamic of herbaceous biomass and epidermal fragments of species from semi-deciduous tropical forest in feces).

En general, la composición bromatológica (tabla III) se localizó dentro de los rangos reportados para el follaje de plantas leñosas de valor forrajero (Pereira et al., 2003; Benezra et al. 2003; Mayer et al., 2003: García et al., 2006), y aunque se presentan diferencias estadísticas $(p<0,05)$ entre especies, todas pueden ser consideradas un buen vehículo de nutrientes al vacuno en silvopastoreo.

El contenido de PB en las 10 leñosas analizadas se situó en 17,5 $\pm 3,4 \%$, siendo superior a pastos tropicales naturales e introducidos quienes muestran en estado juvenil un $12,7 \% \mathrm{~PB}$, y en avanzado estado de madurez un 5,9\% PB (Combellas, 1986). Esto resalta el valor estratégico de los sistemas silvopastoriles tropicales, convirtiéndose en una importante fuente de nitrógeno al vacuno cuando la disponibilidad y calidad de vegetación graminiforme puede resultar limitante a una producción sostenible. La fracción de EE alcanzó 3,1 $\pm 1,0 \%$, lo cual puede ser de interés desde el punto de vista energético a pesar que su valor nutricional no ha sido evaluado en recursos vegetales de este tipo, donde puede existir una importante participación de cubiertas serosas

Tabla III. Composición bromatológica y fracciones de fibra del follaje de especies leñosas del bosque semicaducifolio tropical. (Foliage bromatological composition and fibrous fractions of woody species from semi-deciduous tropical forest).

\begin{tabular}{|c|c|c|c|c|c|c|c|c|c|}
\hline & \multicolumn{9}{|c|}{ Fracciones (\% MS) } \\
\hline & MS & PB & EE & FDN & FDA & Cel & LAD & $\mathrm{Ca}$ & $\mathrm{P}$ \\
\hline R. spinosa & $53,2^{\mathrm{a}}$ & $15,1^{\mathrm{ab}}$ & $4,1^{a}$ & $50,6^{b}$ & $33,3^{c}$ & 20,3 & $13,0^{c}$ & $2,21^{a}$ & $0,13^{b}$ \\
\hline E. cyclocarpus & $38,5^{\mathrm{b}}$ & $22,7^{\mathrm{a}}$ & $3,5^{\mathrm{a}}$ & $59,6^{\mathrm{b}}$ & $28,1^{c}$ & 24,0 & $13,9^{c}$ & $0,42^{\mathrm{c}}$ & $0,14^{b}$ \\
\hline I. laurina & $40,0^{\mathrm{b}}$ & $19,3^{a}$ & $1,7^{b}$ & $70,2^{\mathrm{a}}$ & $50,8^{a}$ & 23,3 & $28,7^{a}$ & $1,03^{b}$ & $0,15^{b}$ \\
\hline M. humboldtianum & $34,3^{b}$ & $18,3^{a}$ & $2,4^{\mathrm{ab}}$ & $59,1^{b}$ & $34,7^{\mathrm{c}}$ & 24,5 & $10,8^{c}$ & $2,77^{\mathrm{a}}$ & $0,16^{b}$ \\
\hline P. lanceolatum & $38,4^{b}$ & $18,4^{\mathrm{a}}$ & $4,1^{\mathrm{a}}$ & $54,1^{\mathrm{b}}$ & $37,8^{\mathrm{c}}$ & 22,1 & $12,8^{\mathrm{c}}$ & $2,05^{a}$ & $0,13^{b}$ \\
\hline S. saman & $35,9^{b}$ & $20,7^{\mathrm{a}}$ & $3,7^{a}$ & $61,4^{b}$ & $40,9^{b}$ & 23,7 & $17,4^{\mathrm{b}}$ & $0,84^{c}$ & $0,14^{b}$ \\
\hline Senna $s p$. & $42,3^{b}$ & $17,4^{\mathrm{ab}}$ & $4,1^{\mathrm{a}}$ & $36,6^{c}$ & $19,2^{d}$ & 22,6 & $7,1^{\mathrm{d}}$ & $2,13^{a}$ & $0,23^{a}$ \\
\hline S. acuta & $35,3^{b}$ & $16,5^{\mathrm{ab}}$ & $3,6^{a}$ & $53,0^{b}$ & $30,3^{c}$ & 21,6 & $13,1^{\mathrm{c}}$ & $1,9^{a}$ & $0,14^{b}$ \\
\hline G. ulmifolia & $38,7^{b}$ & $15,6^{\mathrm{ab}}$ & $3,0^{\mathrm{a}}$ & $54,7^{\mathrm{b}}$ & $31,3^{c}$ & 21,2 & $14,3^{c}$ & $1,99^{a}$ & $0,25^{\mathrm{a}}$ \\
\hline C. caracasana & $39,2^{\mathrm{b}}$ & $10,4^{b}$ & $1,6^{b}$ & $66,7^{b}$ & $50,1^{\mathrm{a}}$ & 22,7 & $20,1^{\mathrm{b}}$ & $1,78^{a}$ & $0,09^{b}$ \\
\hline Error estándar & $5,1^{*}$ & $2,2^{* *}$ & $0,6^{*}$ & $4,1^{* *}$ & $6,3^{*}$ & 3,2 & $1,4^{*}$ & $0,3^{*}$ & $0,04^{*}$ \\
\hline
\end{tabular}

abcd Medias con superíndices desiguales en columnas difieren estadísticamente $\left({ }^{*} p<0,05 ;{ }^{* *} p<0,01\right)$ MS: materia seca; PB: proteína bruta; EE: Extracto etéreo; FDN: fibra detergente neutro; FDA: fibra ácido detergente; Cel: celulosa; LAD: lignina ácido detergente. 
Tabla IV. Fenoles totales, taninos condensados y astringencia del follaje de especies leñosas del bosque semicaducifolio tropical. (Total phenols, condensed tannins and astringency of woody species foliage from semi-deciduous tropical forest).

\begin{tabular}{lccc}
\hline & $\begin{array}{c}\text { Fenoles totales } \\
(\% \text { Eat) }\end{array}$ & $\begin{array}{c}\text { Taninos condensados } \\
(\text { Eleu })\end{array}$ & $\begin{array}{c}\text { Astringencia } \\
\text { (g Eat/100 g MS) }\end{array}$ \\
\hline R. spinosa & $2,0^{\mathrm{c}}$ & $0,33^{\mathrm{c}}$ & $\mathrm{Nd}$ \\
E. cyclocarpus & $3,3^{\mathrm{c}}$ & $0,54^{\mathrm{c}}$ & $\mathrm{Nd}$ \\
I. laurina & $3,7^{\mathrm{c}}$ & $0,44^{\mathrm{c}}$ & $1,18^{\mathrm{b}}$ \\
M. humboldtianum & $2,9^{\mathrm{c}}$ & $0,53^{\mathrm{c}}$ & $\mathrm{Nd}$ \\
P. ligustrinum & $4,7^{\mathrm{bc}}$ & $0,66^{\mathrm{c}}$ & $\mathrm{Nd}$ \\
S. saman & $3,2^{\mathrm{c}}$ & $0,73^{\mathrm{c}}$ & $0,31^{\mathrm{c}}$ \\
Senna sp. & $11,6^{\mathrm{a}}$ & $4,16^{\mathrm{a}}$ & $5,37^{\mathrm{a}}$ \\
S. acuta & $3,0^{\mathrm{c}}$ & $0,55^{\mathrm{c}}$ & $0,20^{\mathrm{c}}$ \\
G. ulmifolia & $2,4^{\mathrm{c}}$ & $0,50^{\mathrm{c}}$ & $\mathrm{Nd}$ \\
C. caracasana & $6,5^{\mathrm{b}}$ & $1,46^{\mathrm{b}}$ & $2,40^{\mathrm{b}}$ \\
Error estándar & $1,22^{* *}$ & $0,41^{*}$ & $1,91^{*}$ \\
\hline
\end{tabular}

${ }^{a b c}$ Medias con superíndices desiguales en columnas difieren estadísticamente $\left({ }^{*} p<0,05 ;{ }^{* *} p<0,01\right)$.

Eat: Equivalentes de ácido tánico ( Lab. Merck $^{\circledR}$ ); Eleu: Equivalentes de leucocianidina. $\mathrm{Nd}=$ No detectado.

totalmente refractarias a la digestión por rumiantes (Van Soest, 1994).

El material evaluado presentó 1,69 \pm $0,16 \% \mathrm{Ca}$ y $0,76 \pm 0,05 \% \mathrm{P}$, valores que comparados con lo referido por Combellas (1986) para pastos tropicales en diferentes estados fenológicos $(0,51 \pm 0,12 \%$ Ca y 0,22 $\pm 0,08 \% \mathrm{P}$ ) enfatizan la superioridad relativa de los follajes de las plantas leñosas en cuanto a su contenido de $\mathrm{Ca}$, con valores que incluso se ajustan a los requerimientos de vacunos con niveles medios de producción (NRC, 2001); aunque los valores de P son inferiores a tales requerimientos, quedando en evidencia la necesidad de implantar programas de suplementación de dicho mineral al animal en silvopastoreo de formaciones boscosas similares.

El contenido de FDN arrojó 57,6 $\pm 11,3 \%$ y el de FDA un $37,1 \pm 12,0 \%$, niveles superiores a lo referido para el follaje de otras especies leñosas de valor forrajero a nivel nacional (García et al., 2006; Baldizán y Chacón, 2007), lo cual además de las diferencias interespecíficas y variaciones en las condiciones ambientales, probablemente se asocie al carácter semicaducifolio del bosque en estudio, donde la elevada vida media de sus hojas obliga al desarrollo de una pared celular de alta resistencia a las condiciones ambientales del medio tropical (Van Soest, 1994). La lignina representó el 15,1 \pm $5,90 \%$ de la fracción fibrosa, valor elevado que puede generar efectos adversos sobre la capacidad de ingestión de alimentos, la digestibilidad de la ración, la síntesis de proteína microbiana ruminal y el aporte de energía (Provenza y Balph, 1988).

Tal como se muestra en la tabla IV, Senna $s p$. y C. caracasana presentaron los mayores contenidos de FT y TC $(\mathrm{p}<0,05)$. Si bien en general los niveles de FT se ubicaron en el espectro referido en la literatura para arbustos y árboles tropicales, la concentración de TC es inferior a lo esperado de acuerdo a los niveles de FT, y a lo citado para plantas leñosas no cultivadas (Makkar, 2001; Frutos et al., 2002; García et al., 2006). Al menos dos causales pueden explicar esta respuesta, en primer lugar una baja partici- 


\section{SELECCIÓN DE ESPECIES LEÑOSAS POR VACUNOS}

pación de leucocianidinas en la estructura química de la fracción de polifenoles, y por tanto una reducida sensibilidad al patrón empleado en el análisis. Por otro lado, el manejo tradicional del pastoreo estacional en estos bosques no promueve la interacción planta-ramoneador, lo que posiblemente imposibilitaría la fijación genética de estrategias fitoquímicas de defensa, tales como la síntesis y almacenamiento vacuolar de TC (Launchbaugh et al., 2001). A pesar de lo anterior, los niveles de TC se localizaron en el rango considerado como beneficioso a la nutrición del rumiante al reducir el riesgo de timpanismo, controlar infecciones parasitarias y promover el flujo de nitrógeno al tracto posterior (Makkar, 2001).

De acuerdo a la clasificación propuesta por Hagerman (1987), sólo la especie Senna $s p$. presentó una elevada cantidad de taninos que precipitan proteína, mientras I. laurina y $C$. caracasana presentaron valores medios para este parámetro. El resto de las especies presentaron valores bajos $(S$. saman y G. ulmifolia) o no detectables por la metodología empleada.

En la tabla V se presentan las correlaciones entre la selectividad del follaje de las plantas leñosas y las variables de la compo- sición química y la astringencia. La selectividad, medida a través del índice de Ivlev (IS), no presentó $(\mathrm{p}>0,05)$ asociación con las variables consideradas en el presente estudio. Por su parte, la FDN estuvo relacionada positivamente con el contenido de LAD, y este a su vez con la FDA. El contenido de FT, TC y la astringencia se presentaron como variables positivamente relacionadas entre si. Estos resultados ponen de manifiesto que, tal como lo señalan Personious et al. (1987), cuando el contenido de compuestos fenólicos no se ubica en niveles limitantes al consumo voluntario, otros factores químicos pueden jugar un rol fundamental en la selectividad del follaje de especies leñosas. En este sentido, García et al. (2008) al evaluar en cabras la preferencia por el follaje de especies forrajeras leñosas con bajo contenido de compuestos fenólicos, concluyen que sólo los metabolitos secundarios del grupo de los isoprenoides (esteroles, terpenos y saponinas) presentaron relación negativa con el consumo voluntario.

El presente estudio es consistente con la tendencia de otros similares, donde se evidencia que los vacunos en silvopastoreo muestran preferencia no sólo por especies

Tabla $\boldsymbol{V}$. Relaciones entre selectividad y variables químicas del follaje de especies leñosas del bosque semicaducifolio tropical. (Relationships among electivity and chemical variables of woody species foliage from semi-deciduous tropical forest).

\begin{tabular}{|c|c|c|c|c|c|c|c|c|}
\hline & PB & FDN & FDA & Cel & LAD & $\mathrm{FT}$ & $\mathrm{TC}$ & Astringencia \\
\hline IS & 0,44 & 0,09 & $-0,05$ & 0,36 & 0,12 & $-0,17$ & $-0,08$ & $-0,05$ \\
\hline PB & & 0,02 & $-0,26$ & 0,54 & $-0,03$ & $-0,19$ & $-0,16$ & $-0,27$ \\
\hline FDN & & & $0,88^{* *}$ & 0,41 & $0,85^{*}$ & $-0,51$ & $-0,64$ & $-0,45$ \\
\hline FDA & & & & 0,18 & $0,87^{*}$ & $-0,29$ & $-0,45$ & $-0,23$ \\
\hline Cel & & & & & 0,17 & 0,11 & 0,05 & 0,06 \\
\hline LAD & & & & & & $-0,28$ & $-0,41$ & $-0,16$ \\
\hline FT & & & & & & & $0,97^{* *}$ & $0,96 * *$ \\
\hline TC & & & & & & & & $0,96^{* *}$ \\
\hline
\end{tabular}

${ }^{*} p<0,05 ;{ }^{* *} p<0,01$.

PB: proteína bruta; FDN: fibra detergente neutro; FDA: fibra ácido detergente, Cel: celulosa, LAD: lignina ácido detergente; FT: fenoles totales; TC: taninos condensados; IS: índice de selectividad de Ivlev. 


\section{OJEDA, OBISPO, CANELONES Y MUÑOZ}

leñosas pertenecientes a la familia de las Fabáceas,sino que incluyen en su dieta una amplia variedad de follajes que en su conjunto representan un importante aporte de nutrientes. Por ello, el diseño de sistemas sustentables de silvopastoreo del bosque tropical debe considerar la promoción de plantas leñosas de valor forrajero, sin afectar la biodiversidad de estos ecosistemas.

\section{CONCLUSIONES}

Las especies leñosas de mayor selectividad para vacunos en silvopastoreo de un bosque semicaducifolio tropical durante la época seca fueron S. acuta, I. laurina, $S$. saman y M. humboldtianum, cuya compo-

\section{BIBLIOGRAFÍA}

AOAC. 1990. Official Methods of Analysis. $15^{\text {th }}$ ed Association of Official Agricultural Chemistry. Washington D.C. USA. 500 pp.

Baldizán, A., Domínguez, C.E., García, D.E., Chacón, E. y Aguilar, L. 2006. Metabolitos secundarios y patrón de selección de dietas en el bosque deciduo tropical de los llanos centrales venezolanos. Zootec Trop, 24: 213-232.

Baldizán, A. y Chacón, E. 2007. Utilización del recurso bosque de los llanos centrales con rumiantes. Memorias. I Simposio Tecnologías Apropiadas para la Ganadería de los Llanos de Venezuela. Universidad Nacional Experimental Rómulo Gallegos. Valle de la Pascua. Venezuela. pp. 79-109.

Barbour, B., Burk, J., Pitts, W., Gilliam, F.S. and Schwartz, W.M. 1999. Terrestrial plant ecology. $3^{\text {rd }}$ ed. Addison-Wesley Longman. Menlo Park, CA. USA. 649 pp.

Benezra, M., Cecconello, G. y Camacho, F. 2003. Selección de especies leñosas en un bosque seco tropical por vacunos adultos usando análisis histológico fecal. Zootec Trop, 21: 73-85.

Combellas, J. 1986. Alimentación de vacas lecheras en el trópico. Lunaprint de Venezuela. Maracay. pp. 146-160.

Fick, K., McDowell, L., Miles, P., Wilkinson, N., Funk, J. y Conrad, J. 1979. Métodos de análisis de minerales para tejidos de plantas y animales. $2^{\mathrm{a}} \mathrm{ed}$. Universidad de Florida. Gainesville. USA. 57 pp. sición química las define como fuente estratégica de nutrientes durante periodos de escasez. En general, el contenido de taninos que precipitan proteínas de las especies seleccionadas puede representar una alternativa para incrementar el flujo de nutrientes al tracto posterior, y con ello, mejorar la respuesta del vacuno en silvopastoreo.

\section{AGRADECIMIENTOS}

Los autores desean expresar su agradecimiento al Consejo de Desarrollo Científico y Humanístico de la Universidad Central de Venezuela por el apoyo financiero para la realización de esta investigación (Proyecto $\left.\mathrm{CDCHN}^{\circ} \mathrm{PG}-01-36-4995-2002\right)$.

Fiske, C. and Subarrow, Y. 1925. The colorimetric determination of phosphorus. J Biol Chem, 66: 375-400.

Frutos, P., Hervás, G., Ramos, G., Giráldez, F.J. and Mantecón, A.R. 2002. Condensed tannin content of several shrub species from a mountain area in northern Spain, and its relationship to various indicators of nutritive value. Anim Feed Sci Tech, 95: 215-226.

García, D.E., Medina, M.G., Humbría, J., Domínguez, C.E., Baldizán, A., Cova, L.J. y Soca, M. 2006. Composición proximal, niveles de metabolitos secundarios y valor nutritivo del follaje de algunos árboles forrajeros tropicales. Arch Zootec, 55: 373-384.

García, D.E., Medina, M.G., Cova, L.J., Humbría, J., Torres, A. y Moratinos, P. 2008. Preferencia caprina por especies forrajeras con amplia distribución en el Estado Trujillo, Venezuela. Arch Zootec, 57: 403-407.

Grela, I. 2003. Evaluación del estado sucesional de un bosque subtropical de quebradas en el norte de Uruguay. Acta Bot Bras, 17: 315-324.

Hagerman, A. 1987. Radial diffusion method for determining tannin plant extracts. J Chem Ecol, 13: 437-449.

Holeckek, J. 1982. Sample preparation technique for microhistological analysis. J Range Manage, 35: 267-268.

Launchbaugh, K.L., Provenza, F.D. and Pfister, 


\section{SELECCIÓN DE ESPECIES LEÑOSAS POR VACUNOS}

J.A. 2001. Herbivore response to anti-quality factors in forages. J Range Manage, 54: 431440.

Lechowicz, M.J. 1982. The sampling characteristics of electivity indices. Oecologia, 52: 22-30.

López, F., Agüero, C., Cruz, A., Rocha, A., Navarrete, N., Flores, G., Kato, E., Sánchez, S., Abarca, L.G. y Bedia, C.M. 1998. Manual de Ecología. Editorial Trillas. $2^{\mathrm{a}}$ ed. México. $112 \mathrm{pp}$.

Makkar, H.P.S. 2001. Quantification of tannins in tree foliage. A laboratory manual for the $\mathrm{FAO} /$ IAEA Co-coordinated Research Project on "Use of Nuclear and Related Techniques to Develop Simple Tannin Assays for Predicting and Improving the Safety and Efficiency of Feeding Ruminants on Tanniferous Tree Foliage". FAO/ IAEA. Viena. 26 pp.

Marquardt, S., Márquez, A., Bouillot, H., Beck, S.G., Mayer, A.C., Kreuzer, M. and Alzérreca, H. 2009. Intensity of browsing on trees and shrubs under experimental variation of cattle stocking densities in southern Bolivia. Forest Ecol Manag, 258: 1422-1428.

Mayer, A.C., Stöckli, V., Huovinen, C., Konold, W., Estermann, B.L. and Kreuzer, M. 2003. Herbage selection by cattle on sub-alpine wood pastures. Forest Ecol Manag, 181: 39-50.

Miliani, T., Espinoza, F., Gil, J.L., Baldizán, A. y Díaz, Y. 2008. Composición botánica de un bosque seco tropical al noreste del estado Guárico, Venezuela. Zootec Trop, 26: 211-214.

Muller-Dumbois, D. and Ellemberg, H. 1974. Aims and methods of vegetation ecology. John Wiley \& Sons. New York. 547 pp.

NRC. 2001. Nutrient requirements of dairy cattle. $7^{\text {th }}$ revised edition. National Research Council. National Academy Press. Washington, D.C. USA. 408 pp.

Pereira, J.A., Quintana, R.D. and Monge, S. 2003. Diets of plains vizcacha, greater rhea and cattle in Argentina. J Range Manage, 56:13-20.

Personious, T.L., Nwambolt, C.L., Stephens, J.R. and Keiser, R.C. 1987. Crude terpenoid influence on mule deer preference for sagebrush. $J$ Range Manage, 40: 84-88.

Porter, L.J., Hrstich, L.N. and Chan, B.G. 1986. The conversion of procyanidins and prodelphinidins to cyanidin and delphinidin. Phytochemistry, 25: 223-230.

Provenza, F.D. and Balph, D.F. 1988. Development of dietary choice in livestock on rangelands and its implications for management. J Anim Sci, 66: 2356-2368.

Rutter, S.M. 2006. Diet preference for grass and legumes in free-ranging domestic sheep and cattle: Current theory and future application. Applied Anim Behaviour Sci, 97: 17-35.

Rosales, M. 1998. Avances en el uso de la diversidad forrajera tropical para la alimentación de bovinos. En: T. Clavero (Ed.). Estrategias de alimentación para la ganadería tropical. Universidad del Zulia. Maracaibo. Venezuela. pp. 85-100. SAS. 1994. User's guide. $4^{\text {th }}$ ed. Statistical Analysis System Institute, Inc. North Carolina, USA. $470 \mathrm{pp}$.

Steel, R. y Torrie, J. 1985. Bioestadística: Principios y procedimientos. $2^{a}$ ed. McGraw-Hill. Bogotá. Colombia. 622 pp.

Terril, T., Windham, W., Evans, J. and Hoveland, C. 1994. Effect of drying method and condenses tannin on detergent fiber analysis of Sericea lespedeza. J Sci Food Agric, 66: 337-343.

Valero, J., Benezra, M.A., Camacaro, C., Chong, L. y Guenni, O. 2005. Identificación botánica y producción de frutos en un bosque deciduo del asentamiento Las Peñitas, al sur del estado Aragua, Venezuela. Zootec Trop, 23: 121-139.

Van Soest, P.J., Robertson, J. and Lewis, B. 1991. Methods for dietary fiber, neutral detergent fiber and non starch polysaccharides in relation to animal nutrition. J Dairy Sci, 74: 3583-3597.

Van Soest, P. 1994. Nutritional Ecology of the Ruminant. Cornell University Press. $2^{\text {th }}$ ed. Ithaca, NY. 476 pp. 